DOI 10.1007/s00417-005-1147-4

\author{
Ute E. K. Schnurrbusch \\ Claudia Jochmann \\ Peter Wiedemann \\ Sebastian Wolf
}

\section{Quantitative assessment of the long-term effect of photodynamic therapy in patients with pathologic myopia}

Received: 20 August 2004

Revised: 2 December 2004

Accepted: 14 January 2005

Published online: 15 March 2005

C) Springer-Verlag 2005
U. E. K. Schnurrbusch · S. Wolf $(\bowtie)$

Klinik und Poliklinik für

Augenheilkunde,

University Bern, Inselspital,

3010 Bern, Switzerland

e-mail: sebastian.wolf@insel.ch

Tel.: +41-31-6328503

C. Jochmann $\cdot$ P. Wiedemann

Klinik und Poliklinik für

Augenheilkunde, University Leipzig,

Liebigstraße 10-14,

04103 Leipzig, Germany
Abstract Background: In patients with classic subfoveal choroidal neovascularization $(\mathrm{CNV})$ secondary to pathologic myopia (PM), photodynamic therapy (PDT) has been shown to stabilize the visual acuity. We have analyzed the long-term visual prognosis after PDT in patients with CNV secondary to PM. Methods: In a retrospective study we reviewed the clinical charts of 102 patients. PDT was performed following the procedures described in the VIP and TAP study protocols. Follow-up examinations and PDT treatment were scheduled in 3-month intervals. Indications for retreatment were an active leaking $\mathrm{CNV}$ in combination with visual disturbances or visual loss. To assess treatment effects we analyzed the number of letters read on the ETDRS charts. The primary efficacy outcome was the proportion of eyes that had fewer than eight letters loss or gain at 24 months. Secondary efficacy outcomes included the proportion of eyes that had fewer than 15 letters loss or gain and the proportion of eyes that had fewer than 30 letters loss or gain at month 24. Results: One hundred and two patients were included into the study. Mean follow-up was $32.5 \pm$ 5.7 months. Patients received an average of 2.2 treatments from study entry through the last follow-up, resulting in a total of 221 PDT sessions throughout the study. At 24 months $46 \%$ lost at least eight letters. A loss of at least 15 (30) letters was observed in $25 \%(8 \%)$. Improvement of at least eight letters was noted in $8 \%$. Larger improvements of at least 15 letters occurred only in $4 \%$ of study eyes. Conclusion: Our study suggests that PDT can increase the chance of stabilizing or improving vision compared with the placebo arm of VIP trial.

Keywords Pathologic myopia . Choroidal neovascularization . Photodynamic therapy

\section{Introduction}

Pathologic myopia (PM) is the most frequent cause of choroidal neovascularization $(\mathrm{CNV})$ in patients under 50 years of age. Approximately $60 \%$ of CNV are secondary to $\mathrm{PM}$ in this age group [4]. PM is characterized by progressive increases in the axial length of the eye and highly negative spherical equivalents (typically $-6.0 \mathrm{dpt}$. or more negative). $\mathrm{CNV}$ develop in $5-10 \%$ of patients with axial length greater than $26.5 \mathrm{~mm}$ [6]. Most of those patients present with classic CNV [15, 17].

The underlying cause of CNV in PM involves degeneration of Bruch's membrane followed by the in-growths of new vessels. Continued stretching and degeneration of the choroid leads to the development of breaks in Bruch's membrane known as lacquer cracks [6]. In eyes with CNV secondary to PM, these have been identified in approximately $80 \%$ of cases $[15,16]$. The presence of these cracks 
allow vessels from the choriocapillaris to proliferate into the subretinal space.

Studies show that although laser photocoagulation may stabilize visual acuity in eyes with extrafoveal CNV secondary to PM $[1,5]$, there is no evidence to suggest benefits in patients with subfoveal CNV $[1,5]$. The studies on laser photocoagulation in extrafoveal lesions also show a high rate of recurrence and a risk of spontaneous progressive enlargement of the atrophic photocoagulation scar leading to worsening of visual acuity [7]. In addition, laser photocoagulation has recently been linked to the development of new or expanded lacquer cracks in the region of the laser scar [8]. In patients with subfoveal CNV secondary to PM, photodynamic therapy (PDT) with verteporfin has been shown to stabilize and even improve visual acuity $[15,16]$, thus providing the means to treat a condition for which no other treatment has been proven effective. However, the 2-year results of the VIP study did not show statistically significant differences between treated and nontreated groups from the primary efficacy outcome [17]. We have analyzed in a retrospective study the long-term visual prognosis after PDT in patients with CNV secondary to PM.

\section{Materials and methods}

We reviewed the clinical charts of 102 patients treated with PDT for subfoveal CNV secondary to PM between 1999 and 2003 in the outpatient department of the University Eye Hospital Leipzig. Inclusion criteria were PM $(-6.0 \mathrm{dpt}$ or more negative), the presence of a classic subfoveal CNV, and a minimal follow-up of 24 months. PDT treatment with verteporfin was performed following the procedures described in the VIP and TAP study protocols. Follow-up examinations were scheduled in 3-month intervals. PDT treatment was performed at baseline and thereafter in 3-month intervals, if indicated. Indications for retreatment were an active leaking $\mathrm{CNV}$ in fluorescein angiographic studies in combination with visual disturbances or visual loss. At each visit all patients underwent a complete eye examination including a detailed medical and ocular history. Examinations included ETDRS visual acuity, binocular ophthalmoscopy, fluorescein angiography with a scanning laser ophthalmoscope (HRA, Heidelberg Eye Explorer, Heidelberg Engineering, Dossenheim, Germany), and color fundus photography. To assess treatment effects we analyzed the number of letters read on the ETDRS charts. The primary efficacy outcome was the proportion of eyes that had fewer than eight letters loss or gain (approximately less than 1.5 lines of visual acuity change corresponding to less than a 1.5-times increase or decrease of the visual angle) at 24 months. Additionally, we analyzed the changes in visual outcome at the last follow-up. Secondary efficacy outcomes included the proportion of eyes that had fewer than 15 letters loss or gain (moderate loss or gain in visual acuity approximately less than three lines of visual acuity change) and the proportion of eyes that had fewer than 30 letters loss or gain (approximately less than six lines of visual acuity change) at month 24 . To assess treatment effects we analyzed the size of the classic component of the $\mathrm{CNV}$ in all angiograms. The size of the classic component of the CNV was evaluated from early angiographic images (30 s) using the tracking tool of the Heidelberg Eye Explorer (Heidelberg Engineering, Dossenheim, Germany). All measurements were performed by two independent readers from the Leipzig Photographic Reading Center with no clinical data available. The study complied with the provisions of the Declaration of Helsinki and the recommendations of the local ethics committee.

\section{Results}

One hundred and two patients ( 37 male, 65 female) aged from 18 to $83(57 \pm 14.8)$ years with classic subfoveal CNV due to PM were included into the study (Table 1). Myopia ranged from $-6.5 \mathrm{dpt}$ to $-25 \mathrm{dpt}$ (mean: $-12.5 \mathrm{dpt}$ ). Followup ranged form 24 to 53 months ( $32.5 \pm 5.7$ months). All patients completed the month 24 examination, and 34 (33\%) patients had a 36 months follow-up. Patients received an average of 2.2 treatments from study entry through the last follow-up, resulting in a total of 221 PDT sessions throughout the study. The average number of applications of verteporfin treatment continued to decrease through the second year from 2.8 by the month- 12 examinations to 0.9 by the month- 24 examinations. At baseline, the main presenting symptom was a decrease in visual acuity. It was associated with metamorphopsia in all patients. Inability to read or drive, loss of stereopsis, altered color vision, and flashes of light were common associated symptoms. Initial visual acuity ranged from 30 to 75 letters (55 \pm 11 letters; approximate Snellen equivalent: 20/80). At the 12-month follow-up examination, visual acuity ranged from 24 to 80 letters (mean 50 \pm 14 letters; approximate Snellen equivalent: 20/100). After 24 months of follow-up, visual acuity ranged from 15 to 79 letters (mean $48 \pm 15$ letters; approximate Snellen equivalent: 20/100). At 36 months and thereafter, the mean visual acuity score was stable at $46 \pm 16$ letters (approximate Snellen equivalent: 20/125).

Table 1 Patient demographics

\begin{tabular}{ll}
\hline Number of patients & 102 \\
Female & 65 \\
Treated eye right & 52 \\
Age (years) & $57.0 \pm 14.8$ \\
Follow up (months) & $32.5 \pm 5.7$ \\
Number of treatments & 221 \\
Number of treatments per eye & $2.2 \pm 0.9$ \\
\hline
\end{tabular}


Table 2 Percentage of changes in visual acuity at the month-24 follow-up examination

\begin{tabular}{lllllll}
\hline & $\begin{array}{l}\geq 30 \\
\text { letters } \\
\text { decrease } \\
(\%)\end{array}$ & $\begin{array}{l}15-29 \\
\text { letters } \\
\text { decrease } \\
(\%)\end{array}$ & $\begin{array}{l}5-14 \\
\text { letters } \\
\text { decrease } \\
(\%)\end{array}$ & $\begin{array}{l} \pm 4 \\
\text { letters } \\
(\%)\end{array}$ & $\begin{array}{l}5-14 \\
\text { letters } \\
\text { increase } \\
(\%)\end{array}$ & $\begin{array}{l}15-29 \\
\text { letters } \\
\text { increase } \\
(\%)\end{array}$ \\
\hline $\begin{array}{l}\text { Current } \\
\text { study } \\
(n=102)\end{array}$ & 8 & 17 & 34 & 25 & 12 & 4 \\
$\begin{array}{l}\text { VIP } \\
\text { placebo } \\
(n=39)\end{array}$ & 8 & 20 & 28 & 31 & 13 & 0 \\
$\begin{array}{l}\text { VIP } \\
\text { visudyne } \\
(n=81)\end{array}$ & 11 & 10 & 25 & 15 & 27 & 12 \\
\hline
\end{tabular}

At 24 months 47 study eyes (46\%) lost at least eight letters (approximately 1.5 Snellen lines) of visual acuity. A loss of at least 15 (30) letters of visual acuity was observed in 25 (eight) study eyes $(25 \% ; 8 \%)$. At last follow-up 46 study eyes $(46 \%)$ presented with a loss of at least eight letters of visual acuity. Improvement of visual acuity of at least eight letters was observed in eight study eyes (8\%). Larger improvements of visual acuity of at least 15 letters occurred only in four study eyes (4\%). A detailed distribution of visual acuity changes in the present study at 24 months is presented in Table 2. Additionally, we included the results from the myopic patients of the VIP Study as historic controls in Table 2 [18]. Figure 1 shows the percentage of study eyes with at least eight letters of visual acuity throughout 24 months at indicated time.

A 36-months follow-up had 34 (33\%) patients. At this time point $18(53 \%)$ study eyes had a decrease of visual acuity of eight letters or more. An improvement in visual acuity of at least eight letters was observed in five study eyes $(15 \%)$. Visual acuity changes at 36 months are presented in detail in Table 3.

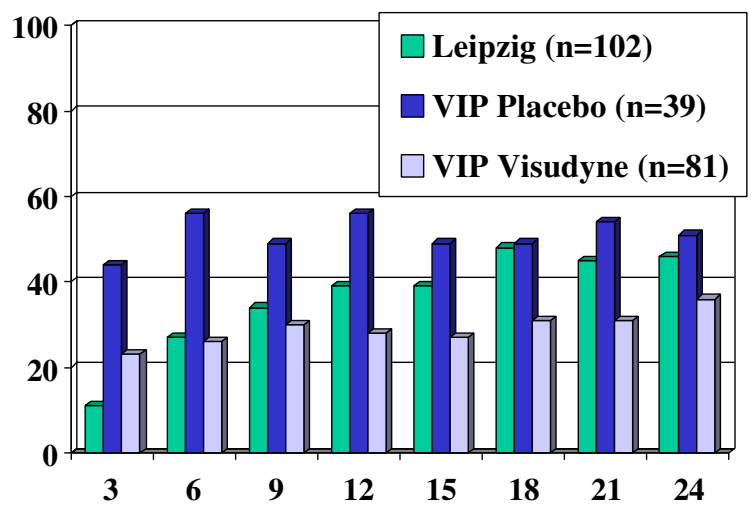

Fig. 1 Percentage with greater than or equal to eight-letter loss at indicated time (data from VIP study $[15,18]$ ).
Table 3 Percentage of changes in visual acuity at the month-36 follow-up examination

\begin{tabular}{|c|c|c|c|c|c|c|}
\hline & $\begin{array}{l}\geq 30 \\
\text { letters } \\
\text { decrease } \\
(\%)\end{array}$ & $\begin{array}{l}15-29 \\
\text { letters } \\
\text { decrease } \\
(\%)\end{array}$ & $\begin{array}{l}5-14 \\
\text { letters } \\
\text { decrease } \\
(\%)\end{array}$ & $\begin{array}{l} \pm 4 \\
\text { letters } \\
(\%)\end{array}$ & $\begin{array}{l}5-14 \\
\text { letters } \\
\text { increase } \\
(\%)\end{array}$ & $\begin{array}{l}\text { 15-29 } \\
\text { letters } \\
\text { increase } \\
(\%)\end{array}$ \\
\hline $\begin{array}{r}\text { Leipzig } \\
(n=34)\end{array}$ & 18 & 26 & 18 & 20 & 12 & 6 \\
\hline
\end{tabular}

The assessment of the classic component of the CNV from early angiographic images showed a size of 0.13 $2.14 \mathrm{~mm}^{2}$ (mean: $0.76 \pm 0.60 \mathrm{~mm}^{2}$ ) at baseline. At the 12-month visit the size of the classic component of the CNV ranged from 0.10 to $9.30 \mathrm{~mm}^{2}$ (mean: $2.0 \pm 2.6 \mathrm{~mm}^{2}$ ) and at 24 months from 0.12 to $9.42 \mathrm{~mm}^{2}$ (mean: $2.0 \pm 2.8$ $\mathrm{mm}^{2}$ ). There were $40(39 \%)$ patients with progression of classic CNV beyond the area of the lesion identified at baseline at the 24-month follow-up. The proportion of lesions with absence of the classic CNV either within or beyond the area of the lesion identified at baseline increased from month 12 with 38 (37\%) patients and to month-24 examination $40(39 \%)$ patients.

\section{Discussion}

In our study we assessed the proportion of patients with subfoveal CNV secondary to PM who developed changes in their visual acuity following PDT. As in the VIP study $[15,18]$, we chose as efficacy outcome the proportion of eyes that had fewer than eight letters loss or gain at 24 months. Since in the TAP study $[13,14]$ the primary outcome was a loss of 15 or more letters of visual acuity loss, we evaluated these changes in visual acuity as well.

The difference between the primary outcome for patients with CNV secondary to PM and secondary to age-related macular degeneration as defined in the VIP and TAP study was chosen because it was expected that eyes with subfoveal CNV due to PM were likely to lose less vision than eyes with subfoveal CNV due to ARMD [15-17].

In previous studies the occurrence of subretinal fibrosis (SRF) after PDT was analyzed in myopic eyes. RuizMoreno and Montero showed that highly myopic eyes with subfoveal CNV treated with PDT have stable visual acuity even through they develop SRF [12]. No correlation between the number of PDT treatments and anatomical and functional results were found in this study [12]. Lanzetta et al. (2004) found several factors for anatomical and functional results such as changes of the retinal pigment epithelium and choroidal vasculature involved in the treatment and enhanced expression of vascular endothelial growth factor [10]. In an other study Montero and Ruiz-Moreno observed an influence of the age at onset but not of the number of PDT treatments on the visual prognosis. This 
was explained with increased chorioretinal atrophy, especially in older patients [11].

In our study the average number of applications of verteporfin treatment continued to decrease through the second year from 2.8 by the month-12 examinations to 0.9 by the month-24 examinations. This low treatment rate compared with the VIP study [15-17] is similar with the current clinical practice in most settings $[2,3,9,11,12]$. Barnes and coworkers proposed that verteporfin therapy can be at least as effective as reported in the literature and requires fewer treatments than reported [2]. Cohen et al. reported a good effect of a single PDT treatment with verteporfin, resulting in involution of the CNV and improvement of visual acuity in a follow-up period of about 17 months [3]. In a prospective study in a Chinese population, visual results were comparable with the VIP study. The average accumulative PDT treatments required in 1 and 2 years were 1.7 and 2.3, respectively [9]. This very low treatment rate compared with the VIP study [17] and our current study could been explained by ethnic differences.

Unlike the VIP investigation, our treatment allocation was not random in nature, and there was no control group. The visual outcome in our study was less favorable compared with the results of the PDT-treated patients in the VIP study. Only during the first 6 months of follow-up was the outcome of our study group similar to the PDTtreated patients in the VIP study. Thereafter, we noted a continuous increase in the number of patients with visual loss in our study. In the VIP study a stabilization of visual acuity loss was noted after the 6-month follow-up. Comparing the number of patients with visual gain of five or more letters, we found similar results. In our study only $16 \%$ of patients had a visual gain compared with $39 \%$ of patients in the PDT-treated patients in the VIP study at the 24-month follow-up. Nevertheless, the number of patients showing an increase in visual acuity is higher than in the placebo arm of the VIP study. Reasons for the less favorable outcome in our study include the relatively low retreatment rate $(2.2 \pm 0.9$ total treatments per eye), the number of patients with excessive myopia ( $>15 \mathrm{dpt}$ ) in our population, and the lower visual acuity at baseline. Especially, the total number of treatments was very low in our population. This may be due in part to the current reimbursement situation for PDT treatment. Since PDT treatment for subfoveal CNV secondary to PM is not covered by public health insurances, most patients were unable to pay for more than one or two PDT treatments. This explains the low number of total treatments in our study.

Looking at the fluorescein angiograms during follow-up, we found a similar rate of patients with progression of classic CNV beyond the area of the lesion identified at baseline (39\% verteporfin-treated group Leipzig vs. 35\% verteporfin-treated group VIP study), but the verteporfintreated groups had a smaller proportion than the placebo treated group in the VIP study (43\%) [17]. The proportion of lesions with absence of the classic CNV either within or beyond the area of the lesion identified at baseline was stable from month 12 to month 24 in $37-39 \%$ of treated patients in our study. In the VIP study the proportion of lesions with absence of classic $\mathrm{CNV}$ either within or beyond the area of the lesion identified at baseline increased from the month-12 (44\%) to the month-24 examination (51\%) [17].

In summary, our study suggests that verteporfin therapy can increase the chance of stabilizing or improving vision compared with the placebo arm of the VIP trial [15-17] in patients with subfoveal CNV caused by PM in a tertiary ophthalmic center. Since most patients with CNV due to PM are young and at employable age, stabilization of visual acuity and eventually restoring of reading ability is extremely important.

\section{References}

1. Avila MP, Weiter JJ, Jalkh AE, Trempe CL, Pruett RC, Schepens CL (1984) Natural history of choroidal neovascularization in degenerative myopia. Ophthalmology 91:1573-1581

2. Barnes RM, Gee L, Taylor S, Briggs MC, Harding SP (2004) Outcomes in verteporfin photodynamic therapy for choroidal neovascularisation-'beyond the TAP study'. Eye 18(8):809-813

3. Cohen SY, Bulik A, Dubois L, Quentel G (2003) Photodynamic therapy for juxtafoveal choroidal neovascularization in myopic eyes. Am J Ophthalmol 136(2):371-374
4. Cohnen SY, Laroche A, Leguen Y, Soubrane G, Coscas GJ (1996) Etiology of choroidal neovascularization in young patients. Ophthalmology 103:1241-1244

5. Fardeau C, Soubrane G, Coscas G (1992) Photocoagulation des néovaisseax sousrétiniens compliquant la dégénérescence myopique. Bull Soc Ophtalmol Fr 92:239-242

6. Hochkiss ML, Fine SL (1981) Pathologic myopia and choroidal neovascularization. Am J Ophthalmol 91:177183

7. Jalkh AE, Weiter JJ, Trempe CL, Pruett RC, Schepens CL (1987) Choroidal neovascularization in degenerative myopia: role of laser photocoagulation. Ophthalmic Surg 18(10):721-725
8. Johnson DA, Yannuzzi LA, Shakin JL, Lightman DA (1998) Lacquer cracks following laser treatment of choroidal neovascularization in pathologic myopia. Retina 18:117-124

9. Lam DS, Chan WM, Liu DT, Fan DS, Lai WW, Chong KK (2004) Photodynamic therapy with verteporfin for subfoveal choroidal neovascularisation of pathologic myopia in Chinese eyes: a prospective series of 1 and 2 year follow up. Br J Ophthalmol 88 (10):1315-1319 
10. Lanzetta P, Battaglia Parodi M, Ambesi-Impiombato M, Ravalico G, Bandello F (2004) Early neovascular bridging after photodynamic therapy of myopic choroidal neovascularization. Graefes Arch Clin Exp Ophthalmol 242(10):840-844

11. Montero JA, Ruiz-Moreno JM (2003) Verteporfin photodynamic therapy in highly myopic subfoveal choroidal neovascularisation. Br J Ophthalmol 87 (2): 173-176

12. Ruiz-Moreno JM, Montero JA (2003) Subretinal fibrosis after photodynamic therapy in subfoveal choroidal neovascularisation in highly myopic eyes. $\mathrm{Br} \mathrm{J}$ Ophthalmol 87(7):856-859
13. TAP Study Group (1999) Photodynamic therapy of subfoveoal choroidal veovascularization in age-related macular degeneration with verteporfin. One-year results of 2 randomized clinical trials-TAP report 1 . Arch Ophthalmol 117:1329-1345

14. TAP Study Group (2001) Photodynamic therapy of subfoveal choroidal neovascularization in age-related macular degeneration with verteporfin: two-year results of 2 randomized clinical trials-TAP report 2. Arch Ophthalmol 119(2):198-207

15. VIP Study Group (2001) Photodynamic therapy of subfoveal choroidal neovascularization in pathologic myopia with verteporfin. 1-year results of a randomized clinical trial-VIP report no. 1. Ophthalmology $108(5)$ : 841-852
16. VIP Study Group (2001) Verteporfin therapy of subfoveal choroidal neovascularization in age-related macular degeneration: two-year results of a randomized clinical trial including lesions with occult with no classic choroidal neovascularization-verteporfin in photodynamic therapy. Report $2 \mathrm{Am}$ J Ophthalmol 131:541-560

17. VIP Study Group (2003) Verteprofin therapy of subfoveal choroidal neovascularization in pathologic myopia. 2year results of a randomized clinical trial-VIP Report No. 3 Ophthalmology 110:666-673

18. VIP-Study Group (2003) Verteprofin therapy of subfoveal choroidal neovascularization in pathologic myopia. 2-year results of a randomized clinical trial-VIP Report No. 3 Ophthalmology 110:666-673 